\title{
Preliminary Numerical Investigation on the Optimization of a Single Bunch of Elastocaloric Elements to be Employed in an Experimental Device
}

\author{
L. Cirillo, A.R. Farina, A. Greco*, C. Masselli \\ DII, University of Naples Federico II, Pl.e Tecchio 80, Naples 80125, Italy
}

Corresponding Author Email: adriana.greco@unina.it

https://doi.org/10.18280/ti-ijes.652-416

Received: 26 March 2021

Accepted: 7 May 2021

\section{Keywords:}

elastocaloric refrigeration, active elastocaloric regenerator, shape memory alloy, numerical model

\begin{abstract}
Nowadays about $20 \%$ of the worldwide energy consumption is attributable to refrigeration almost based on vapor compression. In the scientific literature in the class of the ecofriendly cooling technologies alternative to vapour compression there is solid state cooling. In this field, the scientific community has devoted the attention specifically toward elastocaloric refrigeration. Elastocaloric refrigeration is based on the latent heat associated with the transformation process of the martensitic phase, found in Shape Memory Alloys (SMA) when they are subjected to uniaxial stress cycles of loading and unloading. SMAs are characterized by the mechanical property of being able to return to the initial form once the uniaxial stress has been removed. By exploiting this effect in a reverse regenerative thermodynamic cycle called Active elastocaloric regenerative refrigeration cycle (AeR), a satisfactory cooling effect is achievable. In this paper, the results of a numerical investigation conducted, through a 2-D model, on a single bunch of elastocaloric elements are shown. Specifically, the heat transfer and the energy performances are studied both by varying the geometrical parameters of the elements and by varying the auxiliary fluid (air) velocity.
\end{abstract}

\section{INTRODUCTION}

Montreal [1] and Kyoto Protocol [2], as well as the subsequent Kigali amendment [3], have forced the field of refrigeration and air conditioning towards a sudden removal of refrigerant fluids characterized by high Global Warming Potential (GWP) and Ozone Depletion Potential (ODP) different from zero. On the basis of these directives, the scientific community has moved in search of unconventional refrigeration known as Not-in-Kind [4-6]. Caloric cooling is the most famous between them [7], basing on the phenomenon called caloric effect manifesting in solid-state refrigerants [811]. Solid-state refrigerants have no GWP that result in a more friendly technology with respect to vapor compression [12-14]. Caloric cooling [15] embraces four different technologies: magnetocaloric, electrocaloric, elastocaloric and barocaloric; the name refers to the forcing field that generates the caloric effect. The caloric effect manifest itself in a temperature variation $(\Delta \mathrm{Tad})$ of the solid refrigerant with an adiabatic field variation. When the field increases, a temperature increase is detected; conversely, if the intensity of the field is decreased, a temperature reduction is expected. To a magnetic field variation, a magnetocaloric effect is coupled [16, 17]; electrocaloric cooling derives from the application of an electric field [18]; whereas a mechanical solicitation arises in elastocaloric [19] or barocaloric effect [20,21], respectively if the mechanical field is a structural stress (like traction) or a hydro-static pressure.

The first solid-state technology studied in the last 30 years by scientific community is the magnetocaloric one [22-25]. Gadolinium is the benchmark material of this technology due to its Curie temperature at $294 \mathrm{~K}$, which makes it appropriate for room temperature applications. Nevertheless, over the years more of 100 different experimental devices were developed [26], the commercialization of this technology has not yet reached. The bottleneck lies in the: i) low Coefficients of Performance (COPs), cooling power and temperature span achievable due to the limited $\Delta$ Tad shown by the magnetocaloric refrigerants; ii) low availability of magnetic materials (that almost belong to rare earths); iii) low magnetic field attenable with permanent magnets.

To overcome the above mention limitation, in more recent times scientific community has moved the research on other caloric effect-based cooling techniques. Among them, the most promising seems to be the elastocaloric cooling because of: easiness in applying the mechanical field; cheap and easy to find materials; great mechanical field variation attainable. Binary alloy of Ni-Ti [27-29], are the benchmark Shape Memory Alloys (SMA) with elastocaloric properties at room temperature. Shape Memory is the property of reminding the shape of the material before the application of the uniaxial stress and the capability to recover that shape. The elastocaloric effect observed in SMA is correlated to the latent heat absorbed/released by the material depending on the adiabatic application/remotion of the mechanical field (loading/unloading). During the loading process, the elastocaloric material changes its structural phase passing from Austenite to Martensite (A-M); M-A transformation occurs during unloading. To A-M and $\mathrm{M}-\mathrm{A}$ shape transformations are connected two characteristics of the elastocaloric materials: the shape-memory and the superelasticity. In superelasticity, the SMA can be loaded to very large strains (up to 8.5\%) and release the transformation heat. Upon being unloaded, it can regain its initial shape (without any permanent deformation) 
and absorb the heat. Occurrence of shape-memory effect or superelasticity in a particular SMA sample depends on its temperature. Above austenite stability temperature (Af), a SMA sample is superplastic and below martensite stability temperature (Mf), the sample exhibits shape-memory behaviour.

The elastocaloric materials represent a very incident factor in the realization of the elastocaloric device, as well as on the related energy performance. The role of benchmark of such technology has given to NiTi binary alloy because of the giant adiabatic temperature change shown at room temperature. The elastocaloric materials can be classified in: Shape Memory Alloys (SMA) and in Shape Memory Polymers (SMP).

The SMA class can be further divided in Ni-Ti-based (doped with $\mathrm{Cu}, \mathrm{Co}, \mathrm{Pd}$, etc.), Cu-based (doped with $\mathrm{Al}, \mathrm{Ni}$, $\mathrm{Zn}, \mathrm{Mn}$, etc.) and Fe-based based (doped with Pd, Rh, Mn, Si, $\mathrm{Ni}$, etc.) alloys [30]. Nickel-Titanium alloys can be binary, ternary or quaternary. With respect to the binary, ternary alloys with the addition of vanadium or aluminum to the alloy helps to reduce the stress hysteresis and to enhance fatigue life [31, 32]. The further addendum of a fourth element, like copper, allows to shift the operative temperature range where the Austenite Martensite transformation occurs [33, 34]. Among the $\mathrm{Cu}$-based elastocaloric alloys the attentions goes on the $\mathrm{Cu}-\mathrm{Zn}-\mathrm{Al}$ [35], $\mathrm{Cu}-\mathrm{Al}-\mathrm{Mn}$ [36] ternary the presence of Aluminum ensures an enhancement of the heat exchange coefficients. Under the complete austenite/martensite transformation, $\mathrm{Cu}-\mathrm{Zn}-\mathrm{Al}$ exhibits $15 \mathrm{~K}$ as maximum adiabatic temperature change but the small fatigue life strongly limits the possibility of being employed for applications where long-time operation is needed. About Febased SMAs one can say that the interest on them is more marginal because, always considering under the complete austenite/martensite transformation, much lower than the two previous mentioned classes are both the latent heat and consequently the $\Delta \operatorname{Tad}(5 \mathrm{~K})$, even if a smaller hysteresis is detected [37].

The SMP is a class embracing the so-called soft materials like rubber or polymers [38-40]. As general consideration, these materials are cheaper than Ni-Ti alloys as well as improved is the fatigue life; the major limit is represented by the much larger elongations (volume variations) needed to make the elastocaloric effect manifesting (up to 600\%) that make them not to easy to be employed in experimental devices.

Before to realize an experimental device, it is needed to optimize such aspects and the easy way to perform this task it to operate with a numerical model. The developing of a model able to reproduce the behavior of the experimental prototype is a helpful tool to be guided in realizing the prototype under optimized conditions.

Elastocaloric cooling is a promising technology but many steps are still needed to be taken before it could assume a central role in the refrigeration and air conditioning sector. The prototypes developed are still too few and none are already suitable to be placed on the market. In this scenario Italy has not yet introduced to the scientific community any elastocaloric prototypes suitable for refrigeration/air conditioning. To remedy this aspect, University of Naples Federico II got the challenge to build a demonstrative prototype of an elastocaloric system as the goal of the SUSSTAINEBLE research project. The prototype will be rotary and composed by several bunches of elastocaloric wires crossed by air as heat transfer fluid. Each bunch of the prototype experiments cyclically the four steps of the Active elastocaloric Regenerative cycle. The regenerator (made of elastocaloric SMA) is located between a Cold and a Hot Heat Exchanger (CHEX and HHEX). The four processes are:

(1) adiabatic loading: the stress $\sigma$ is applied to load the SMA, the A-M transformation occurs and the elastocaloric effect derives in a temperature increment in the SMA, equal to $\triangle \mathrm{Tad}$ $(\mathrm{T}, \sigma)$;

(2) the air flow is blown from the from the cold to the hot end of the regenerator and cools the SMA elements at maximum strain. The hot air releases the absorbed heat in the HHEX;

(3) adiabatic unloading: the stress $\sigma$ is removed and the SMA elements cool down for the elastocaloric effect;

(4) the air flow is blown from hot to cold side with no applied stress. The elastocaloric wires cool the air flow. The cold air absorbs heat from the cold heat exchanger producing the cooling effect.

There are many aspects to be optimized:

- the number of the wires and the total number of bunches mounted, as well as the total mass of the elastocaloric refrigerant;

- the dimensional parameters of the bunch as well as the ones of each wire: the diameter, the length, the distance between two of them;

- $\quad$ the working parameters like air velocity and flow rate, the frequency of the elastocaloric cycle;

- the composition of the elastocaloric material and correlatedly the stress applied to the wires.

This paper introduces a two-dimensional model of a single bunch of elastocaloric wires based on finite-element method.

In this paper we report the results of an exhaustive and extensive test campaign focused on the optimization of the crucial parameters like: cycle frequency, air velocity, length of the wires and distance among them.

\section{THE MATHEMATICAL MODEL}

The optimization is focused on a single bunch of NiTi wires that will be the elements that will constitute the active regenerator of the first Italian elastocaloric prototype. The elastocaloric prototype that we intend to design will be in continuous operation, based on rotation mechanism. The experimental setup is based on the mechanical concept of two co-rotating disks with multiple bunches of elastocaloric elements placed around the circumference. We intend to develop the elastocaloric cooler prototype as a direct aircooling unit, avoiding additional heat exchangers. Therefore, the secondary fluid in the prototype will be air that exchanges heat with the elastocaloric elements in forced convective flow. The air will flow in the channel between the two rotating disks investing the elastocaloric elements in parallel direction. An appropriate arrangement of the air inlets and outlets on the cylinder base allows the continuous extraction of hot and cold air.

The first step of the design of the experimental prototype is to focus the attention on a single bunch of wires to optimize the geometrical parameter of the wires and of the bunch, and to choose the most suitable operating parameters such as: frequency of rotation, air velocity, wires length, distance between wires to maximize the heat transfer between air and elastocaloric elements. In this primary investigation the elastocaloric material is the benchmark Ni50.4Ti49.6. The Austenite finish temperature Af is $20^{\circ} \mathrm{C}$, that guarantees the 
achievement of pseudoelastic behavior at room temperature. For this material, the maximum latent heat of the complete Austenite-Martensite transformation is $20 \mathrm{~J} \mathrm{~g} \mathrm{~g}^{-1}$ that corresponds to a maximum adiabatic temperature change of 40 $\mathrm{K}$. The SMA was subjected to a moderate strain rate fixed at $0.25 \mathrm{~s}-1$ (6\% maximum strain) that corresponds to a time of around $200 \mathrm{~ms}$ for each loading and unloading process. Higher strain-rates corresponding to higher operating frequencies would also be possible, but to increase the fatigue life the strain-rate has been identified with the lower value of the adiabatic limit. The operative frequency of the cycle has been changed in the simulations by varying the fluid blow period.

The mathematical model that governs the AeR cycle has been formulated according to the following assumptions:

i. the time required for loading and unloading the SMA elements is negligible compared with the heat transfer time with the air flow. Therefore, both the processes can be considered adiabatic.

ii. the loading/unloading processes occur uniformly though uniaxially stretching operations;

iii. the regenerator is considered adiabatic with the surrounding;

iv. the model is two-dimensional;

v. the elastocaloric effect is evaluated through theestimation of the latent heat and the work needed for loading/unloading following the Austenite-Martensite or Martensite-Austenite transformations.

The system of the equations governing the model is:

$$
\left\{\begin{array}{c}
\frac{\partial u}{\partial x}+\frac{\partial v}{\partial y}=0 \\
\frac{\partial u}{\partial t}+u \frac{\partial u}{\partial x}+v \frac{\partial u}{\partial y}=-\frac{1}{\rho_{f}} \frac{\partial p}{\partial x}+v_{f}\left(\frac{\partial^{2} u}{\partial x^{2}}+\frac{\partial^{2} u}{\partial y^{2}}\right) \\
\frac{\partial v}{\partial t}+u \frac{\partial v}{\partial x}+v \frac{\partial v}{\partial y}=-\frac{1}{\rho_{f}} \frac{\partial p}{\partial y}+v_{f}\left(\frac{\partial^{2} v}{\partial x^{2}}+\frac{\partial^{2} v}{\partial y^{2}}\right) \\
\rho_{f} c_{f} \frac{\partial T_{f}}{\partial t}+\rho_{f} c_{f}\left(u \frac{\partial T_{f}}{\partial x}+v \frac{\partial T_{f}}{\partial y}\right)=k_{f}\left(\frac{\partial^{2} T_{f}}{\partial x^{2}}+\frac{\partial^{2} T_{f}}{\partial y^{2}}\right) \\
\rho_{S M A} c_{S M A} \frac{\partial T_{S M A}}{\partial t}=k_{S M A}\left(\frac{\partial^{2} T_{S M A}}{\partial x^{2}}+\frac{\partial^{2} T_{S M A}}{\partial y^{2}}\right)+\mathrm{G}
\end{array}\right.
$$

where $\mathrm{G}$ is the elastocaloric term that depends both on the latent heat and the loading/unloading work that can be vealuated as:

$$
G=\rho_{S M A}(\Delta H+w) \dot{\xi}_{M}
$$

In shape-memory alloys the amount of latent heat associated to the forward and reverse austenite-martensite transformations is different for the hysteresis curve, as well as a different value assumes the work required for loading/unloading. According to Tusek et al. [42] the net-work is:

$$
w \text { net }=w_{\text {load }}-w_{\text {unload }}
$$

representing the area enclosed by the entropy-temperature hysteresis cycle.

The volume fraction of the martensitic phase $\xi$ can estimated through the differential equation (4):

$$
\dot{\xi}_{M}=\xi_{M} \psi^{M A}\left(T_{S M A}, \sigma\right)-\xi_{A} \psi^{A M}\left(T_{S M A}, \sigma\right)
$$

where,

$$
\xi_{M}+\xi_{A}=1
$$

Since the time required for the A-M M-A transformations to take place is negligible, the term $\xi$ only converts itself in +1 or -1 , i.e. $G$ changes its sign during the loading/unloading processes. During the convective heat transfer process, the stress applied is not varying, thus $\xi=0$ and the elastocaloric term $\mathrm{G}$ is zero.

\section{OPERATING CONDITIONS}

For the design of the experimental device the relevant parameters influencing the energy and thermal performances must be identified. To this aim the numerical model is used for optimization. The bunch of elastocaloric elements, representing the active regenerator, is supposed to be in contact in both the convective heat transfer phase steps with external air at $293 \mathrm{~K}$. For loading the SMA elements at a strain rate of $0.25 \mathrm{~s}-1,0.2 \mathrm{~s}$ is the time of each loading/unloading step. The operative frequency of the cycle has been changed in the simulations by varying the fluid blow period.

According to Ossmer et al. [41] the following values of the properties of Ni50.4Ti49.6 have been used: a thermal conductivity of $15 \mathrm{~W} \mathrm{~m}^{-1} \mathrm{~K}^{-1}$; a density of $6500 \mathrm{~kg} \mathrm{~m}^{-3}$; a specific heat of $500 \mathrm{~J} \mathrm{~kg}^{-1} \mathrm{~K}^{-1}$.

To maximize the cycle frequency, the flow blowing time should be a multiple of $\tau$ that is the transient constant that regulates the convective heat exchange between the air flow and the SMA wire:

$$
\tau=\frac{\rho_{S M A} c_{S M A} V_{\text {wire }}}{h A_{\text {wire }}}=\frac{\rho_{S M A} c_{S M A} D_{\text {wire }}}{4 h}
$$

This equation clearly shows that to maximize the cycle frequency the diameter of the wire should be minimized. In these simulations a diameter of $0.3 \mathrm{~mm}$ was chosen because it was not considered appropriate to choose a smaller diameter toavoid problems related to the breaking of the wires into the device.

The fluid blow time (always a multiple of) was tested according to the following values: $t_{f}=[1.0 ; 2.0 ; 4.0 ; 6.0] \mathrm{s}$.

The values of the air velocity have been selected according to typical values of the air conditioning systems: $\vec{v}=[3.0 ; 5.0 ; 7.0 ; 9.0 ; 11.0] \mathrm{ms}^{-1}$.

Different distances among the wires were tested: $\boldsymbol{d}=[0.5$; $1 ; 2] \mathrm{mm}$.

The length of the wires was investigated among the following values: $\boldsymbol{L}=[100 ; 200 ; 300] \mathrm{mm}$.

To evaluate the energy performances, the temperature span and the AeR cooling power, defined respectively in Eq. (7) and (8), were calculated after each test:

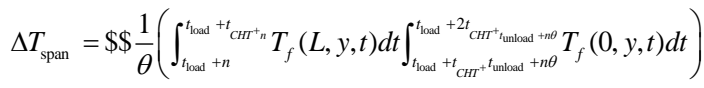

The temperature span is defined as the difference between the average temperature of the hot air leaving the regenerator in the second step of the cycle (when the heat is released) and the temperature of the cold air exiting from the regenerator in the fourth step of the cycle. 


$$
\dot{Q}_{\text {ref }}=\frac{1}{\theta} \int_{t_{\text {toad }}+t_{f}+t_{\text {unload }}+n \theta}^{t_{\text {toad }}+*^{*}+t_{t}+t_{\text {ullax }}+n \theta} \dot{m}_{f} C_{f}\left(T_{\text {env }}-T_{f}(0, y, t)\right) d t
$$

The cooling power accounts the integral in the time of the difference between environmental temperature $(293 \mathrm{~K})$ and the temperature of the temperature of the cold air exiting from the regenerator in the fourth step of the cycle.

\section{RESULTS AND DISCUSSION}

Figure 1 report the temperature span as a function of the cycle frequency parametrized for air velocity when the distance between two wires is: (a) $0.5 \mathrm{~mm}$; (b) $1.0 \mathrm{~mm}$; (c) 2.0 $\mathrm{mm}$. Figures clearly show that for each fluid velocity and distance between wires there is an optimal frequency that maximizes the temperature span. The lower is the air flow speed, the smaller is the value of the optimal frequency. This is due to the higher values of convective heat exchange transient constant required for smaller velocity, since greater is the time for optimal heat exchange. At fixed velocity, if the distance between two wires is greater, the optimal frequency point is observed for a higher value.

For each distance the same trend can be observed: increasing the air velocity the temperature span decreases. This decrease is more evident at low cycle frequencies. The mean value of this decrease is around $-40 \%$. The reason of such behaviour is that air flows with lower velocities result in a longer time at which the air is in contact with elastocaloric wire: consequently, the heat transfer increases with reducing air velocity.

A smaller distance between two wires ensures the achievement of higher temperature spans $(+50 \%$ as medium increment on the $\Delta$ Tspan values calculated for $\mathrm{d}=0.5 \mathrm{~mm}$ with respect to $\mathrm{d}=1.0 \mathrm{~mm}$ ). This behavior is more pronounced at higher air velocity (the mean increment is $40 \%$ at $3 \mathrm{~m} \mathrm{~s}^{-1}$ air velocity and $60 \%$ at $11 \mathrm{~m} \mathrm{~s}^{-1}$ ). Indeed, decreasing distance between two wires at fixed inlet air velocity increases the air velocity between the wires and therefore the convective heat transfer coefficient, enhancing the heat transfer between the air flow and the wire wall.
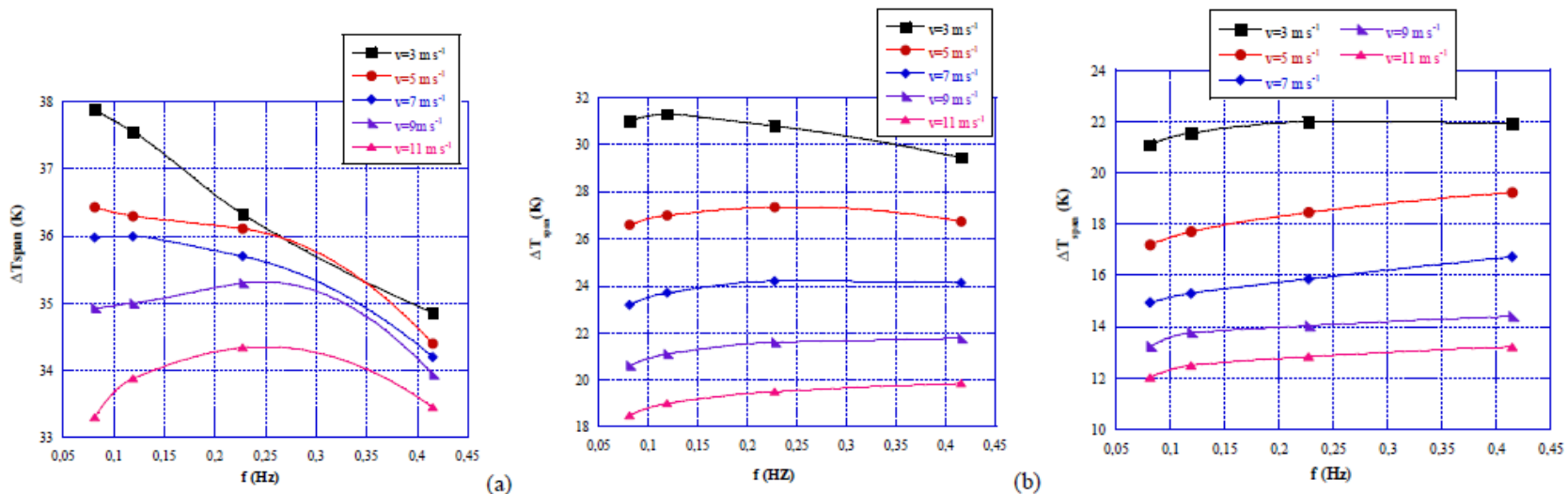

Figure 1. Temperature span as a function of the cycle frequency parametrized for air velocity when the distance between two wires is: (a) $0.5 \mathrm{~mm}$; (b) $1.0 \mathrm{~mm}$; (c) $2.0 \mathrm{~mm}$
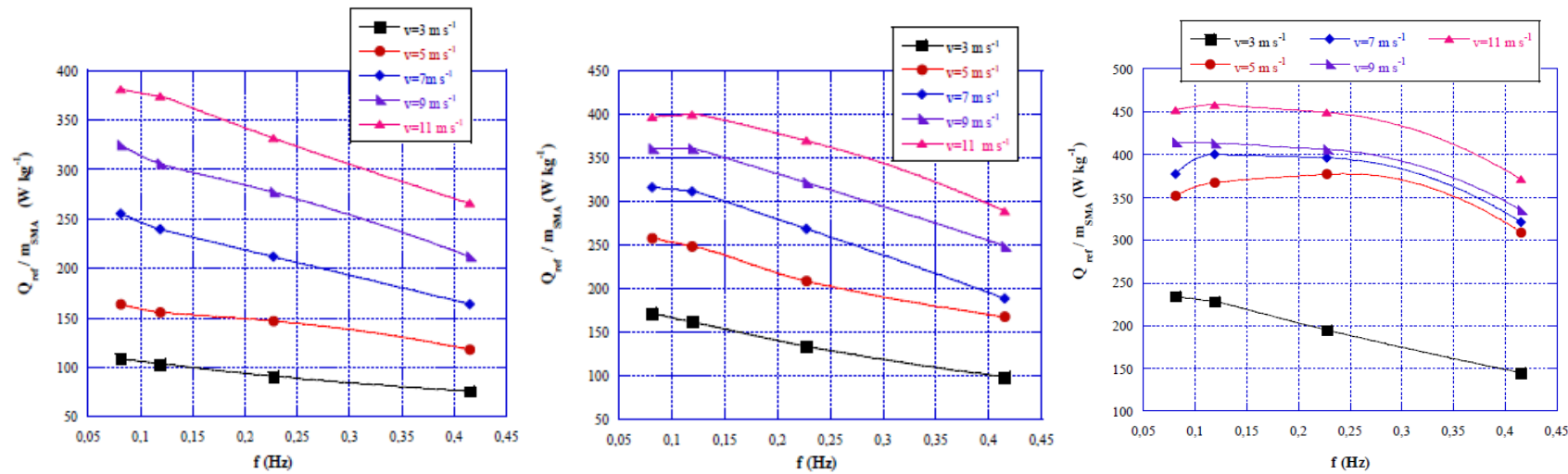

Figure 2. Cooling power per unit of SMA mass as a function of the cycle frequency parametrized for air velocity when the distance between two wires is: (a) $0.5 \mathrm{~mm}$; (b) $1.0 \mathrm{~mm}$; (c) $2.0 \mathrm{~mm}$

Figure 2 reports the cooling power per mass unit of SMA as a function of the cycle performance parametrized for fluid velocity when the distance between two wires is: (a) $0.5 \mathrm{~mm}$; (b) $1.0 \mathrm{~mm}$; (c) $2.0 \mathrm{~mm}$.

Figure 2 clearly shows that at each distance the greater is the velocity of the air, the larger is the cooling power. The mean increment is $+60 \%$ (between 3 and $11 \mathrm{~m} \mathrm{~s}^{-1}$ ). Indeed, increasing the air velocity the heat transfer coefficient increases, enhancing the heat transfer between the air flow and the elastocaloric material. This increment is more marked for the lower distance between the wires (the mean increment is + $70 \%$ at $\mathrm{d}=0.5 \mathrm{~mm}$ whereas is $50 \%$ at $\mathrm{d}=2 \mathrm{~mm}$ ). At fixed air velocity the cooling power is maximum in correspondence of a specific optimal frequency. On equal operative conditions, the optimal frequency where the maximum in cooling power occurs is different from the optimal frequency, ensuring a 
maximum in temperature span.

The figures clearly show that increasing the distance between the wires the cooling power per unit mass also increases. The increase is due to the increase of the air flowing between the wires (at fixed number of wires). This increment is more marked al low air flow. Specifically, one can observe that for lower velocities, for example at $3 \mathrm{~m} \mathrm{~s}^{-1}$, the geometry configuration where the wires are placed at $1.0 \mathrm{~mm}$ or $2.0 \mathrm{~mm}$ as a distance, gives respectively $+50 \%$ and $+40 \%$ as medium cooling power per mass unit augmentation with respect to $\mathrm{d}=$ $0.5 \mathrm{~mm}$; if the speed increases up to $11 \mathrm{~m} \mathrm{~s}^{-1}$, the power gain is reduced until reaching $+8 \%$ and $+18 \%$, respectively for 1.0 $\mathrm{mm}$ and $2.0 \mathrm{~mm}$.

These results clearly show that the existence of a trade-off between the maximization of temperature span and the maximization of cooling power. Therefore, the most suitable solution would be to operate with values located in the middle of the range choosing $1.00 \mathrm{~mm}$ as optimal distance between wires. Fixed the wire distance at $1 \mathrm{~mm}$, the length of the wires has been investigated. Figure 3 report the curves of the temperature span as a function of the cycle frequency parametrized for air velocity when the length of the wires is: (a) $100 \mathrm{~mm}$; (b) $200 \mathrm{~mm}$; (c) $300 \mathrm{~mm}$.

From the Figure 3 one can notices that for slower velocity of the air flow higher values of temperature span are registered. The augmentation of the length of the wire has a positive effect on $\Delta \mathrm{T}$ span, even if the values of percentage increment with the length are strongly dependent on the air velocity, more than cycle frequency. Specifically, with air velocity at $3 \mathrm{~m} \mathrm{~s}^{-1}$ a medium value (with respect to the tested frequencies) of $+7 \%$ on $\Delta$ Tspan is registered if the length of the wire passes from $100 \mathrm{~mm}$ to $300 \mathrm{~mm}$, whereas the same medium increment arises up to $+69 \%$ if the air velocity is $11 \mathrm{~m} \mathrm{~s}^{-1}$. Furthermore, the effect of the length augmentation on $\Delta$ Tspan from $200 \mathrm{~mm}$ to $300 \mathrm{~mm}$ is as more evident as the velocity increases whereas it is completely inexistent for $3 \mathrm{~m} \mathrm{~s}^{-1}$.

The differences between the absolute maximum and the absolute minimum, proper of each data set are: $12.7 \mathrm{~K}$ for $\mathrm{L}=100 \mathrm{~mm} ; 7.3 \mathrm{~K}$ for $\mathrm{L}=200 \mathrm{~mm} ; 4.4 \mathrm{~K}$ for $\mathrm{L}=300 \mathrm{~mm}$. Indeed, the lower is the length of the NiTi wires, the most sensitive is the geometry configuration to the variation of the main parameters under investigation: air velocity and cycle frequency.
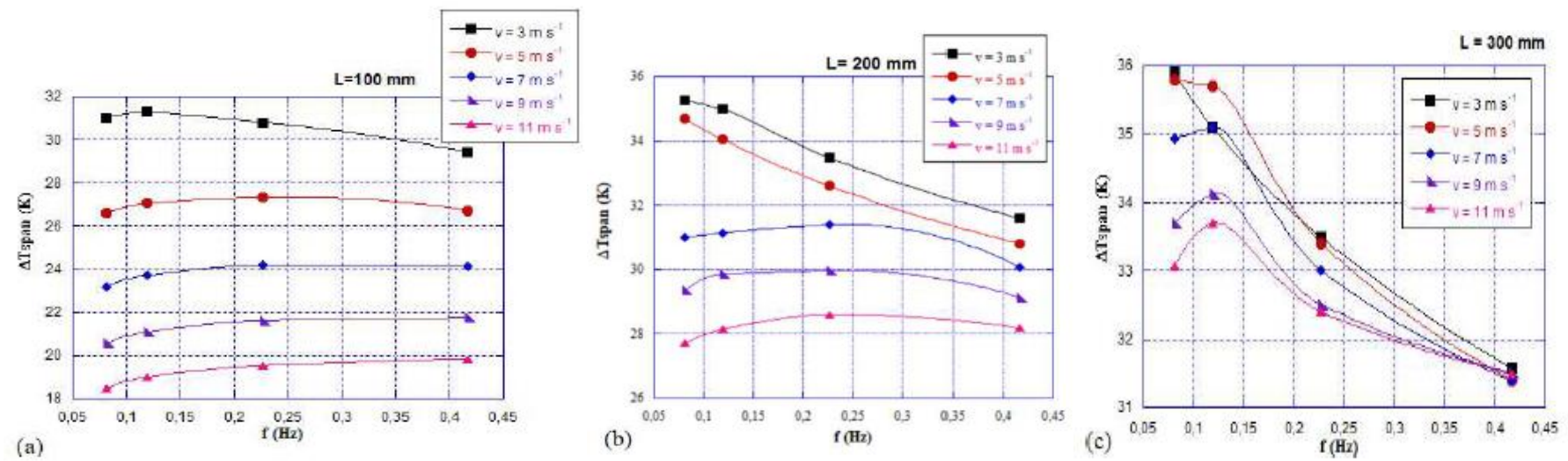

Figure 3. Temperature span as a function of the cycle frequency parametrized for air velocity when the length of the wires is: (a) $100 \mathrm{~mm}$; (b) $200 \mathrm{~mm}$; (c) $300 \mathrm{~mm}$
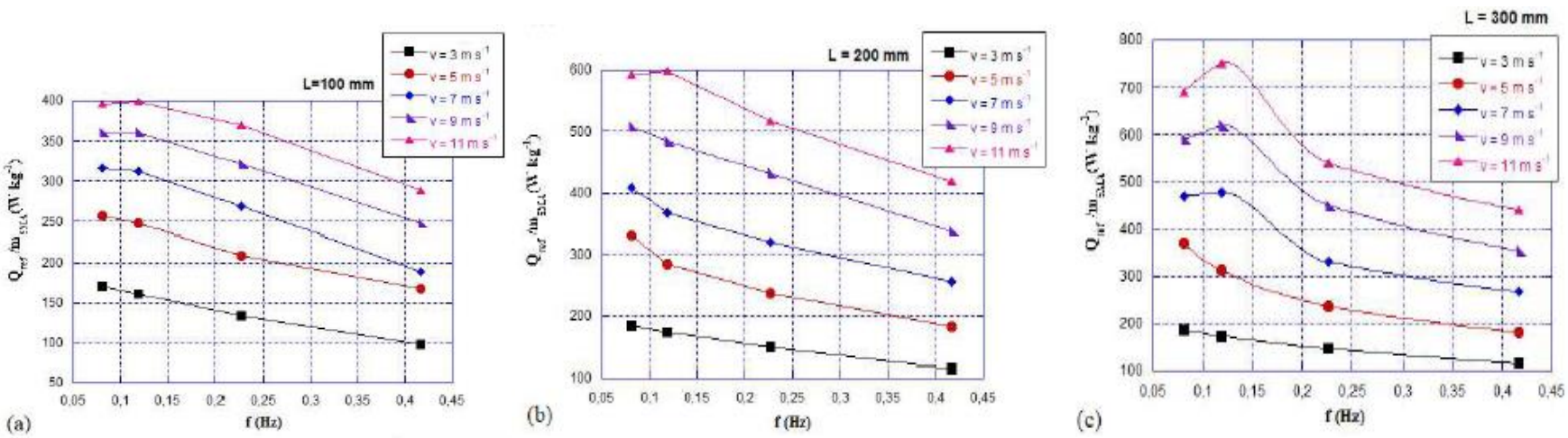

Figure 4. Cooling power per mass unit as a function of the cycle frequency parametrized for air velocity when the length of the wires is: (a) $100 \mathrm{~mm}$; (b) $200 \mathrm{~mm}$; (c) $300 \mathrm{~mm}$

Figure 4 report the cooling power per mass unit as a function of the frequency parametrized for fluid velocity when the length of the wires is: (a) $100 \mathrm{~mm}$; (b) $200 \mathrm{~mm}$; (c) $300 \mathrm{~mm}$. From Figure 4 the overall consideration is that the greater is the velocity of the air, the larger is the cooling power. The optimal frequency that is not the same for all the lengths. Moreover, on equal operative conditions, the optimal frequency where the maximum in cooling power occurs is different from the optimal frequency ensuring a maximum in temperature span. The cooling power per unit mass increases also with the length of the wire: indeed, the heat exchange paths that the air travels are the longer, the bigger is the wire length; it derives in an increment of the heat exchange area. The effect of the length augmentation on cooling power is always very pronounced if L grows from $100 \mathrm{~mm}$ to $200 \mathrm{~mm}$, for all the air velocities and the frequencies tested. On the contrary for a length increment from $200 \mathrm{~mm}$ to $300 \mathrm{~mm}$ the effect of the increase in cooling power derives in a saturation 
that manifests for all the frequencies if the velocity is $3 \mathrm{~m} \mathrm{~s}^{-1}$. For greater speeds, the effect of the saturation is visible only from the frequency $0.227 \mathrm{~Hz}$ upwards.

At $3 \mathrm{~m} \mathrm{~s}^{-1}$, the effect of increasing the length from $100 \mathrm{~mm}$ to $200 \mathrm{~mm}$ brings $+10.5 \%$ as medium rise in cooling power, whereas there is no increment if $\mathrm{L}$ passes from $200 \mathrm{~mm}$ to 300 $\mathrm{mm}$. At $11 \mathrm{~m} \mathrm{~s}^{-1}$, the medium augmentations are: $+45.4 \%$ from $100 \mathrm{~mm}$ to $200 \mathrm{~mm}$ and $+12.8 \%$ from $200 \mathrm{~mm}$ to $300 \mathrm{~mm}$.

\section{CONCLUSIONS}

The attention in this paper has been devoted to the optimization of the frequency of the cycle and the air flow velocity with respect to variable lengths and distance of the wires of a bunch of $\mathrm{Ni}$-Ti that will be one of the active elements to be placed in the first Italian elastocaloric prototype. The bunches will be arranged circularly, and they will be crossed by an air flow. The aim is to ensure the heat exchange between heat transfer fluid and SMA would occur as more optimized as possible with the finale objective to maximize both the temperature span and the cooling power. By the analysis of the results the following considerations can be drawn:

- $\quad$ for all the investigated lengths of the wires and for every frequency the temperature span decreases with the augmentation of the air velocity;

- $\quad$ at fixed air flow velocity, there is an optimal frequency that maximizes the temperature span. On equal velocity values, the optimal frequency decreases if the wire length increases and if the distance between the wires decreases.

- A smaller distance between two wires ensures the achievement of higher temperature spans $(+50 \%$ as medium increment on the $\Delta$ Tspan values calculated for $\mathrm{d}=0.5 \mathrm{~mm}$ with respect to $\mathrm{d}=1.0 \mathrm{~mm}$ ).

- for every air velocity and cycle frequency, the augmentation of the wire length from $100 \mathrm{~mm}$ to $200 \mathrm{~mm}$ has a positive effect on $\Delta$ Tspan. The effect of the length augmentation on $\Delta$ Tspan from $200 \mathrm{~mm}$ to $300 \mathrm{~mm}$ is as more evident as the velocity increases whereas it is completely inexistent for $3 \mathrm{~m} \mathrm{~s}^{-1}$.

- The cooling power is greater as greater are the values of air velocity. Indeed, a trade-off in maximizing temperature span and cooling power exists.

- On equal air flow velocity and cycle frequency, there is a growth of the cooling power with the increasing of the distance between two wires (i.e. with the increasing of the air flow rate).

- The cooling power per mass unit, on equal other conditions, increases also with the length of the wire. This effect is always very pronounced if $\mathrm{L}$ grows from $100 \mathrm{~mm}$ to $200 \mathrm{~mm}$, for all the air velocities and the frequencies tested. On the contrary for a length increment from $200 \mathrm{~mm}$ to $300 \mathrm{~mm}$, the effect of the increase in cooling power derives in a saturation that manifests for all the frequencies if the velocity is $3 \mathrm{~m} \mathrm{~s}^{-1}$. For greater speed the effect of the saturation is visible only from the frequency $0.227 \mathrm{~Hz}$ upwards.

In conclusion, with respect to the cycle frequency and the air flow velocity, given the revealed trade-off between the maximization of temperature span and cooling power, in our opinion the most suitable solution would be to operate with values located in the middle of the ranges. Specifically, the choice of: i) $0.119 \mathrm{~Hz}$ as cycle frequency; ii) $7 \mathrm{~m} \mathrm{~s}^{-1}$ as air flow velocity; iii) $1.0 \mathrm{~mm}$ as optimal distance to place the wires; iv) $200 \mathrm{~mm}$ as wire length.

\section{ACKNOWLEDGEMENTS}

This research was funded through the project "SUSSTAINEBLE" - FISR2019 04798 granted by FISR Fondo Integrativo Speciale per la Ricerca (Italian special supplementary fund for research).

\section{REFERENCES}

[1] Protocol, M. (1987). Montreal protocol on substances that deplete the ozone layer. Washington, DC: US Government Printing Office, 26: 128-136.

[2] Breidenich, C., Magraw, D., Rowley, A., Rubin, J.W. (1998). The Kyoto protocol to the United Nations framework convention on climate change. American Journal of International Law, 92(2): 315-331. https://doi.org/10.2307/2998044

[3] Heath, E.A. (2017). Amendment to the Montreal protocol on substances that deplete the ozone layer (Kigali amendment). International Legal Materials, 56(1): 193205. https://doi.org/10.1017/ilm.2016.2

[4] Renaldi, R., Miranda, N.D., Khosla, R., McCulloch, M.D. (2021). Patent landscape of not-in-kind active cooling technologies between 1998 and 2017. Journal of Cleaner Production, 296: 126507. https://doi.org/10.1016/j.jclepro.2021.126507

[5] Qian, S., Nasuta, D., Rhoads, A., Wang, Y., Geng, Y., Hwang, Y., Radermacher, R., Takeuchi, I. (2016). Notin-kind cooling technologies: A quantitative comparison of refrigerants and system performance. International Journal of Refrigeration, 62: 177-192. https://doi.org/10.1016/j.ijrefrig.2015.10.019

[6] Brown, J.S., Domanski, P.A. (2014). Review of alternative cooling technologies. Applied Thermal Engineering, 64(1-2): 252-262. https://doi.org/10.1016/j.applthermaleng.2013.12.014

[7] Kitanovski, A., Plaznik, U., Tomc, U., Poredoš, A. (2015). Present and future caloric refrigeration and heatpump technologies. International Journal of Refrigeration, $\quad 57$ : 288-298. https://doi.org/10.1016/j.ijrefrig.2015.06.008

[8] Moya, X., Mathur, N.D. (2020). Caloric materials for cooling and heating. Science, 370(6518): 797-803. https://doi.org/10.1126/science.abb0973

[9] Hess, T., Maier, L.M., Bachmann, N., Corhan, P., Schäfer-Welsen, O., Wöllenstein, J., Bartholomé, K. (2020). Thermal hysteresis and its impact on the efficiency of first-order caloric materials. Journal of Applied Physics, 127(7): 075103. https://doi.org/10.1063/1.5132897

[10] Crossley, S., Mathur, N.D., Moya, X. (2015). New developments in caloric materials for cooling applications. Aip Advances, 5(6): 067153. https://doi.org/10.1063/1.4922871

[11] Moya, X., Kar-Narayan, S., Mathur, N.D. (2014). Caloric materials near ferroic phase transitions. Nature Materials, 13(5): 439-450. https://doi.org/10.1038/nmat3951

[12] Greco, A., Vanoli, G.P. (2006). Experimental two-phase pressure gradients during evaporation of pure and mixed 
refrigerants in a smooth horizontal tube. Comparison with correlations. Heat and Mass Transfer, 42(8): 709725. https://doi.org/10.1007/s00231-005-0020-7

[13] Greco, A., Vanoli, G.P. (2005). Flow boiling heat transfer with HFC mixtures in a smooth horizontal tube. Part II: Assessment of predictive methods. Experimental Thermal and Fluid Science, 29(2): 199-208. https://doi.org/10.1016/j.expthermflusci.2004.03.004

[14] Greco, A., Mastrullo, R., Palombo, A. (1997). R407C as an alternative to R22 in vapour compression plant: An experimental study. International Journal of Energy Research, 21(12): 1087-1098 https://doi.org/10.1002/(SICI)1099-

114X(19971010)21:12<1087::AID-ER330>3.0.CO;2-Y

[15] Aprea, C., Greco, A., Maiorino, A., Masselli, C. (2020). The employment of caloric-effect materials for solidstate heat pumping. International Journal of Refrigeration, 109: 1-11. https://doi.org/10.1016/j.ijrefrig.2019.09.011

[16] Aprea, C., Greco, A., Maiorino, A., Masselli, C. (2016). A comparison between different materials in an active electrocaloric regenerative cycle with a $2 \mathrm{D}$ numerical model. International Journal of Refrigeration, 69: 369382. https://doi.org/10.1016/j.ijrefrig.2016.06.016

[17] Aprea, C., Greco, A., Maiorino, A. (2013). The use of the first and of the second order phase magnetic transition alloys for an AMR refrigerator at room temperature: a numerical analysis of the energy performances. Energy Conversion and Management, 70: 40-55. https://doi.org/10.1016/j.enconman.2013.02.006

[18] Aprea, C., Greco, A., Maiorino, A., Masselli, C. (2016). A comparison between different materials in an active electrocaloric regenerative cycle with a $2 \mathrm{D}$ numerical model. International Journal of Refrigeration, 69: 369382. https://doi.org/10.1016/j.ijrefrig.2016.06.016

[19 Qian, S., Geng, Y., Wang, Y., Ling, J., Hwang, Y., Radermacher, R., Cui, J. (2016). A review of elastocaloric cooling: Materials, cycles and system integrations. International Journal of Refrigeration, 64: 1-19. https://doi.org/10.1016/j.ijrefrig.2015.12.001

[20] Aprea, C., Greco, A., Maiorino, A., Masselli, C. (2020). The use of barocaloric effect for energy saving in a domestic refrigerator with ethylene-glycol based nanofluids: A numerical analysis and a comparison with a vapor compression cooler. Energy, 190: 116404. https://doi.org/10.1016/j.energy.2019.116404

[21] Lloveras, P., Tamarit, J.L. (2021). Advances and obstacles in pressure-driven solid-state cooling: A review of barocaloric materials. MRS Energy \& Sustainability, pp. 1-13. https://doi.org/10.1557/s43581-020-00002-4

[22] Gschneidner Jr, K.A., Pecharsky, V.K., Pecharsky, A.O., Zimm, C.B. (1999). Recent developments in magnetic refrigeration. In Materials Science Forum, Trans Tech Publications Ltd, 315: 69-76. https://doi.org/10.4028/www.scientific.net/MSF.315317.69

[23] Yu, B.F., Gao, Q., Zhang, B., Meng, X.Z., Chen, Z. (2003). Review on research of room temperature magnetic refrigeration. International Journal of Refrigeration, 26(6): 622-636. https://doi.org/10.1016/S0140-7007(03)00048-3

[24] Kitanovski, A., Egolf, P.W. (2010). Innovative ideas for future research on magnetocaloric technologies. International Journal of Refrigeration, 33(3): 449-464. https://doi.org/10.1016/j.ijrefrig.2009.11.005
[25] Kitanovski, A. (2020). Energy applications of magnetocaloric materials. Advanced Energy Materials, 10(10): 1903741 . https://doi.org/10.1002/aenm.201903741

[26] Greco, A., Aprea, C., Maiorino, A., Masselli, C. (2019). A review of the state of the art of solid-state caloric cooling processes at room-temperature before 2019. International Journal of Refrigeration, 106: 66-88. https://doi.org/10.1016/j.ijrefrig.2019.06.034

[27] Ossmer, H., Chluba, C., Krevet, B., Quandt, E., Rohde, M., Kohl, M. (2013). Elastocaloric cooling using shape memory alloy films. In Journal of Physics: Conference Series, 476(1): 012138.

[28] Cui, J., Wu, Y., Muehlbauer, J., Hwang, Y., Radermacher, R., Fackler, S., Takeuchi, I. (2012). Demonstration of high efficiency elastocaloric cooling with large $\Delta \mathrm{T}$ using NiTi wires. Applied Physics Letters, 101(7): 073904. https://doi.org/10.1063/1.4746257

[29] Zhou, M., Li, Y.S., Zhang, C., Li, L.F. (2018). Elastocaloric effect and mechanical behavior for NiTi shape memory alloys. Chinese Physics B, 27(10): 106501.

[30] Bruederlin, F., Bumke, L., Chluba, C., Ossmer, H., Quandt, E., Kohl, M. (2018). Elastocaloric cooling on the miniature scale: A review on materials and device engineering. Energy Technology, 6(8): 1588-1604. https://doi.org/10.1002/ente.201800137

[31] Zeng, K., Schmid-Fetzer, R., Huneau, B., Rogl, P., Bauer, J. (1999). The ternary system Al-Ni-Ti part II: Thermodynamic assessment and experimental investigation of polythermal phase equilibria. Intermetallics, 7(12): 1347-1359. https://doi.org/10.1016/S0966-9795(99)00055-2

[32] Kabirifar, P., Žerovnik, A., Ahčin, Ž., Porenta, L., Brojan, M., Tušek, J. (2019). Elastocaloric Cooling: State-of-theart and Future Challenges in Designing Regenerative Elastocaloric Devices. Strojniski Vestnik/Journal of Mechanical Engineering, 65.

[33] Al Hasan, N.M., Hou, H., Sarkar, S., Thienhaus, S., Mehta, A., Ludwig, A., Takeuchi, I. (2020). Combinatorial synthesis and high-throughput characterization of microstructure and phase transformation in $\mathrm{Ni}-\mathrm{Ti}-\mathrm{Cu}-\mathrm{V}$ quaternary thin-film library. $\quad$ Engineering, 6(6): 637-643. https://doi.org/10.1016/j.eng.2020.05.003

[34] Mosca, H.O., Bozzolo, G., Del Grosso, M.F. (2012). Atomistic modeling of ternary additions to $\mathrm{NiTi}$ and quaternary additions to $\mathrm{Ni}-\mathrm{Ti}-\mathrm{Pd}, \mathrm{Ni}-\mathrm{Ti}-\mathrm{Pt}$ and $\mathrm{Ni}-\mathrm{Ti}-$ Hf shape memory alloys. Physica B: Condensed Matter, 407(16): https://doi.org/10.1016/j.physb.2011.12.077

[35] Mañosa, L., Jarque-Farnos, S., Vives, E., Planes, A. (2013). Large temperature span and giant refrigerant capacity in elastocaloric $\mathrm{Cu}-\mathrm{Zn}-\mathrm{Al}$ shape memory alloys. Applied Physics Letters, 103(21): 211904. https://doi.org/10.1063/1.4832339

[36] Yuan, B., Zhu, X., Zhang, X., Qian, M. (2019). Elastocaloric effect with small hysteresis in bamboograined $\mathrm{Cu}-\mathrm{Al}-\mathrm{Mn}$ microwires. Journal of Materials Science, 54(13): 9613-9621. https://doi.org/10.1007/s10853-019-03592-8

[37] Xiao, F., Fukuda, T., Kakeshita, T., Jin, X. (2015). Elastocaloric effect by a weak first-order transformation associated with lattice softening in an Fe-31.2 Pd (at.\%) 
alloy. Acta $\quad$ Materialia, 87: 8-14. $\mathrm{m}^{\cdot} \quad$ flow rate, $\mathrm{kg} \mathrm{s}^{-1}$ https://doi.org/10.1016/j.actamat.2015.01.004 n number of times

[38] Patel, S., Chauhan, A., Vaish, R., Thomas, P. (2016). Elastocaloric and barocaloric effects in polyvinylidene di-fluoride-based polymers. Applied Physics Letters, 108(7): 072903. https://doi.org/10.1063/1.4942000

[39] Xie, Z., Sebald, G., Guyomar, D. (2017). Comparison of elastocaloric effect of natural rubber with other caloric effects on different-scale cooling application cases. Applied Thermal Engineering, 111: 914-926. https://doi.org/10.1016/j.applthermaleng.2016.09.164

[40] Xie, Z., Sebald, G., Guyomar, D. (2015). Elastocaloric effect dependence on pre-elongation in natural rubber. Applied Physics Letters, 107(8): 081905. https://doi.org/10.1063/1.4929395

[41] Ossmer, H., Lambrecht, F., Gültig, M., Chluba, C., Quandt, E., Kohl, M. (2014). Evolution of temperature profiles in TiNi films for elastocaloric cooling. Acta Materialia, 81:

9-20. https://doi.org/10.1016/j.actamat.2014.08.006

[42] Tušek, J., Engelbrecht, K., Mañosa, L., Vives, E., Pryds, N. (2016). Understanding the thermodynamic properties of the elastocaloric effect through experimentation and modelling. Shape Memory and Superelasticity, 2(4): 317-329. https://doi.org/10.1007/s40830-016-0094-8

\section{NOMENCLATURE}

\section{Roman symbols}

$\begin{array}{ll}\text { A } & \text { area, } \mathrm{m}^{2} \\ \text { c } & \text { specific heat capacity, } \mathrm{J} \mathrm{kg}^{-1} \mathrm{~K}^{-1} \mathrm{D} \\ \mathrm{D} & \text { Distance between two wires, } \mathrm{mm} \mathrm{f} \\ \mathrm{G} & \text { elastocaloric term, } \mathrm{KJ} \mathrm{m}^{-3} \\ \mathrm{H} & \text { Latent heat, } \mathrm{J} \mathrm{g}^{-1} \\ \mathrm{~h} & \text { convective heat transfer coefficient, } \mathrm{W} \mathrm{m} \mathrm{m}^{-2} \mathrm{~K}^{-1} \mathrm{k} \\ \mathrm{L} & \text { length of the wire, } \mathrm{mm} \\ \mathrm{m} & \text { Mass, } \mathrm{kg}\end{array}$

